\title{
Analysis of The Global Posture of College Students With Chronic Neck Pain
}

\author{
Jiugen Zhong \\ Shanghai University of Sport \\ Wenhao Wang \\ Guangzhou College of Applied Science and Technology \\ Ligen $\mathrm{Yu}$ \\ Guangzhou Sport University \\ Xiaohui Hou ( $\square$ houxh@gzsport.edu.cn ) \\ Guangzhou Sport University
}

\section{Research Article}

Keywords: Chronic neck pain, global posture, kinematic chain

Posted Date: December 2nd, 2021

DOI: https://doi.org/10.21203/rs.3.rs-1089151/v1

License: (1) (1) This work is licensed under a Creative Commons Attribution 4.0 International License. Read Full License 


\section{Abstract}

Background: Chronic neck pain (CNP) is common, but methods that focus on the cervical spine have not met the patients' medical expectations.

Objective: To investigate the global postural difference between students with CNP and healthy people.

Design: Cross-sectional study.

Methods: Twenty-seven healthy college students without neck pain and 31 students with CNP were recruited and allocated into a control group and a CNP group. Differences in standing postural indicators between the two groups were compared.

Results: Compared to the control group, the leg length discrepancy and the right rearfoot angle were larger and the anterior lower limb alignment angle was smaller. In the sagittal plane, the left sagittal lower limb alignment and right cervical alignment angles were larger, while the left and right sagittal body alignment angles in the CNP group were smaller. The odds ratio calculation for the trunk forward lean, right foot valgus, and knee flexion on both sides indicated that these are risk factors for CNP, while knee varus is not a risk factor for CNP. The remaining abnormal postures were shown not to be associated with CNP.

Limitation: This study did not conduct in-depth research on the physiological state of the muscles, joints, and other structures, and we did not apply these theories to practice.

Conclusions: Abnormal posture in students with CNP is mainly concentrated in the sagittal plane. Trunk forward lean, foot valgus, and knee flexion on both sides are risk factors for CNP.

\section{Introduction}

Chronic neck pain (CNP) is a common condition that not only leads to considerable pain and disability, but it also affects the mental state, reduces work efficiency and quality of life, and represents a significant medical burden. For this reason, CNP has become a public health and social issue ${ }^{[1,2]}$. However, to date, the effect of modern medical methods to relieve neck pain and restore neck function in CNP are unsatisfactory. These methods include neck suspenders, analgesic drugs, physical therapy, exercise ${ }^{[3,4]}$, manipulation ${ }^{[5-8]}$, acupuncture ${ }^{[9,10]}$, massage ${ }^{[11]}$, and surgery. In other words, these methods, which focus on the cervical spine, have not met the patient's medical expectations. Neck pain may occur as a result of imbalances in the biomechanics of the neck and abnormalities in the entire alignment throughout the body. Recent studies have shown that global posture re-education is an effective treatment for chronic pain, including neck and low back pain ${ }^{[12-14]}$. Meanwhile, some studies have provided evidence that ergonomic assistive devices are useful for correcting poor posture ${ }^{[15]}$. Interestingly, these results are in line with the human fascia and muscle chain theory proposed by Myers. 
According to Myers' "Anatomy Trains - The Myofascial Meridian for Manual and Movement Therapy" [16], the skeletal muscle system of the human body is a tensile structure. Thus, from a global posture and kinematic chain viewpoint, there must be a connection between the appearance of local symptoms and overall changes in global posture and kinematic chains. Indeed, when a part of the body's tension changes, it will inevitably cause changes in the tension structure of other parts ${ }^{[16]}$. Similarly, Dr. Janda, a clinical medical scientist from the Czech Republic, proposes three chains responsible for adaptive changes in a certain part of the body from the perspective of clinical assessment and treatment of pain caused by muscle imbalance, namely, the joint, muscle, and neural chains ${ }^{[17]}$. A kinematic chain reaction, also known as the "wave effect," occurs in the entire movement chain of the human body. The primary movement of a joint can produce up, down, left, or right body adjustments. In other words, when a part of the body is under abnormal pressure, other parts of the body also produce abnormal pressure and tension $[18,19]$. Deviations within these three chain systems lead to compensatory and adaptive changes in the neuromuscular system. For example, changes in the joint chain affect the function of the muscle and nerve chains, resulting in a corresponding structure and function of the entire kinematic chain. The result of this change is a dysfunctional movement pattern that occurs throughout the body during static standing or exercise, with significant compensatory movements or joint pain. Some studies have found that head extension and rounded shoulder posture in patients with CNP is more prominent than in a healthy population, and that the greater the degree of head extension, the more serious the dysfunction [19]. However, another study showed that forward head posture (FHP) did not differ significantly between groups nor correlate with muscle performance or CNP clinical characteristics ${ }^{[20]}$.

Whether the imbalance of the global posture is a risk of CNP remains unclear. We hypothesize that there is a potential mechanical imbalance or "weakened chain" in the global posture of patients with CNP. The Global Postural Systems (GPS) Lab (model GPS400, Chinesport, Italy) was used to measure global postural indicators of a healthy and CNP population to explore the connections between local structural changes in the neck and global posture, and provide new insights for treating CNP.

\section{Methods}

This cross-sectional study collected data on college students with and without CNP. The GPS Lab, a professional and reliable assessment tool ${ }^{[21]}$, was used to measure posture.

\section{Participants}

Fifty-eight college students were recruited from five universities in Guangzhou city. Thirty-one students were enrolled in the CNP group, and 27 students without neck pain during the same period were recruited as the control group. All of the participants provided their informed consent.

The inclusion criteria of the CNP group were as follows: 1) recurrent neck pain (Visual Analogue Scale [VAS] $\geq 3$ ) for more than 3 months; and 2) no history of spinal or trauma surgery. The inclusion criteria of the control group were as follows: 1 ) no history of neck pain in the past 3 years; 2 ) no structural 
abnormalities in the neuromusculoskeletal system and no balance disorders; 3 ) no history of sports injury or trauma; and 4) no skeletal muscle pain and/or limited joint range of motion in the past week. Subjects with rheumatism, rheumatoid, gout, mandatory spondylitis, tumors, osteoporosis, fractures, intervertebral disc herniation with nerve compression, or vertebral spondylolisthesis, or those who were pregnant, alcoholics, or drug-dependent were excluded.

\section{Ethics}

Ethics approval of this study was obtained from Guangzhou Sport University (ETHICS No. 2018LCLL-12). An information sheet was provided to all participants prior to enrolment of the study. Written informed consent was obtained from all of the participants.

Neck pain and functional indicators

A VAS score was used to measure the intensity of neck pain. Neck dysfunction was measured using the Neck Disability Index (NDI). Cervical spine mobility of participants was measured in the flexion, extension, lateral flexion, and rotation using a cervical mobility meter (Cervical Test, model 01618, Chinesport, Italy). The muscle strength of the neck muscle groups was tested with portable muscle strength and joint activity meter (MicroFET 3 MT \& ROM, model MicroFET 3, Hoggan Scientific, USA).

Indicators of global posture included the vertical body position, sagittal alignment of the body, sagittal lower limb alignment, shoulder angle, cervical angle, sagittal head angle, rearfoot angle, anterior lower limb alignment, quadriceps angle ( $\mathrm{Q}$ angle), bilateral anterior superior iliac spine (ASIS) height difference, bilateral posterior superior iliac spine (PSIS) height difference, distance of bilateral ASIS to the midline, distance of bilateral PSIS to the midline, horizontal alignment of the ASISs, horizontal alignment of the PSISs, alignment of the scapula related to T3, difference in the alignment of the scapula related to T3, scapular alignment, horizontal alignment of the scapula, horizontal alignment of the acromion, horizontal alignment of the head, inclination of the head, footprint, and difference in length of both lower limbs. The methods for evaluating each global postural indicator are described in Table 1 and Figures 1-3. 
Table 1

Evaluation method and normal range of global posture evaluation indicators

\begin{tabular}{|llll}
\hline Indicators & Normal range & Evaluation method & Figure \\
\hline $\begin{array}{l}\text { Vertical body } \\
\text { position }\end{array}$ & $0.9^{\circ} \sim 2.7^{\circ}$ & $\begin{array}{l}\text { Angle between acromion, the lateral malleolus } \\
\text { line and the plumb line }\end{array}$ & Figure $1 \angle 1$ \\
\hline
\end{tabular}

Sagittal alignment $183.6^{\circ} \sim 190^{\circ}$

Angle between the acromion, the large

Figure $1 \angle 2$

of the body

trochanter of the femur, and the lateral

malleolus

$\begin{array}{lll}\text { Sagittal lower } & 0^{\circ} \sim 5^{\circ} & \begin{array}{l}\text { Angle between the large trochanter of the } \\ \text { femur, the articular line of the knee, and the } \\ \text { lateral malleolus }\end{array}\end{array}$

$\begin{array}{lll}\text { Inclination of the } \quad 7^{\circ} \sim 17^{\circ} & \begin{array}{l}\text { Angle between the ASIS, the PSIS, and a } \\ \text { horizontal line }\end{array} & \text { Figure } 1 \angle 4 \\ \text { Pelvic } & & \end{array}$

Figure $1 \angle 3$

Shoulder angle $\quad 122^{\circ} \sim 142.8^{\circ}$

Angle between the acromions, $\mathrm{C} 7$ and the

Figure $1 \angle 5$

horizontal line

\begin{tabular}{|c|c|c|}
\hline cal Angle & $47.4^{\circ} \sim 56.4^{\circ}$ & $\begin{array}{l}\text { Angle between the C7 spinal process, the ear } \\
\text { lobe, and a horizontal line }\end{array}$ \\
\hline
\end{tabular}

\begin{tabular}{llll}
$\begin{array}{l}\text { Sagittal Head } \\
\text { Angle }\end{array}$ & $0^{\circ} \sim 10^{\circ}$ & $\begin{array}{l}\text { angle between the tragus, the external } \\
\text { canthus of the eye and the horizontal line }\end{array}$ & Figure $\angle 7$ \\
$\begin{array}{l}\text { Rearfoot angle } \\
171^{\circ} \sim 179^{\circ}\end{array}$ & $\begin{array}{l}\text { The Angle between } 1 / 3 \text { of the lower extremity, } \\
\text { the midpoint of the Achilles tendon, and the } \\
\text { midpoint of the bone }\end{array}$ & Figure $\angle 8$ \\
\hline
\end{tabular}

Anterior lower

$174.5^{\circ} \sim 181.7^{\circ}$

Angle between ASIS, the lateral midpoint of

the knee joint and the lateral malleolus tip

Figure3 $\angle 9$

limbs alignment

M: $8.2^{\circ} \sim 14.2^{\circ}$

Angle between ASIS, the mid-point of the

Figure $\angle 10$

Quadriceps angle

F: $11.3^{\circ} \sim 20.3^{\circ}$

patella and trochanter of the tibia

$0.5 \mathrm{~cm}$

Perpendicular to the reference line, compare

the two sides and calculate the difference

Figure3 $I_{1}$

bilateral ASIS

height

$\begin{array}{llll}\begin{array}{l}\text { Difference of } \\ \text { bilateral PSIS } \\ \text { height }\end{array} & 0.5 \mathrm{~cm} & \begin{array}{l}\text { Perpendicular to the reference line, compare } \\ \text { the two sides and calculate the difference }\end{array} & \text { Figure } 2 \mathrm{I}_{2} \\ & & \end{array}$

\section{Distance of bilateral ASIS to midline}

Distance of bilateral PSIS to midline
$0.5 \mathrm{~cm}$

$0.5 \mathrm{~cm}$

The difference is calculated by comparing the horizontal distance between the two sides and the midline

The difference is calculated by comparing the horizontal distance between the two sides and the midline
Figure $3 \mathrm{I}_{3}$

Figure $3 \mathrm{I}_{4}$

Note: LL:lower limbs;ASIS=anterior superior iliac spine; PSIS=posterior superior iliac spine; M: man; F: female. 


\begin{tabular}{|c|c|c|c|}
\hline Indicators & Normal range & Evaluation method & Figure \\
\hline $\begin{array}{l}\text { Horizontal } \\
\text { alignment of the } \\
\text { ASISs }\end{array}$ & $-1.4^{\circ} \sim 2.2^{\circ}$ & $\begin{array}{l}\text { Angle between the } 2 \text { ASISs and a horizontal } \\
\text { line }\end{array}$ & $\begin{array}{l}\text { Figure } \\
3 \angle 11\end{array}$ \\
\hline $\begin{array}{l}\text { Horizontal } \\
\text { alignment of the } \\
\text { PSISs }\end{array}$ & $-1.3^{\circ} \sim 3.1^{\circ}$ & $\begin{array}{l}\text { Angle between the } 2 \text { PSISs and a horizontal } \\
\text { line }\end{array}$ & Figure3 $\angle 12$ \\
\hline $\begin{array}{l}\text { Alignment of the } \\
\text { scapula related to } \\
\text { T3 }\end{array}$ & $/ /$ & $\begin{array}{l}\text { Difference of the distances of the scapulas to } \\
\text { the T3 vertebrae }\end{array}$ & Figure2 $I_{5}$ \\
\hline $\begin{array}{l}\text { Difference in } \\
\text { alignment of the } \\
\text { scapulas related } \\
\text { to T3 }\end{array}$ & $0.5 \pm 1.1 \mathrm{~cm}$ & $\begin{array}{l}\text { The distance between the medial margin of } \\
\text { the scapula and the spine of the third thoracic } \\
\text { spine, and the difference of the distance } \\
\text { between the two sides }\end{array}$ & Figure $2 I_{5}$ \\
\hline $\begin{array}{l}\text { Scapulars } \\
\text { alignment }\end{array}$ & $1.8^{\circ} \sim 11.4^{\circ}$ & $\begin{array}{l}\text { Angle between the point of intersection of the } \\
\text { spine of the scapula and the medial margin, } \\
\text { the inferior angle of the scapula, and a } \\
\text { horizontal line }\end{array}$ & Figure $2 \angle 13$ \\
\hline $\begin{array}{l}\text { Horizontal } \\
\text { alignment of the } \\
\text { scapulas }\end{array}$ & $-3.9^{\circ} \sim 4.7^{\circ}$ & $\begin{array}{l}\text { Angle between the inferior angles of the } \\
\text { scapulas and a horizontal line }\end{array}$ & Figure $2 \angle 14$ \\
\hline $\begin{array}{l}\text { Horizontal } \\
\text { alignment of the } \\
\text { acromion }\end{array}$ & $0.8^{\circ} \sim 3.2^{\circ}$ & $\begin{array}{l}\text { Angle between the two-sided acromions line } \\
\text { and the horizontal line }\end{array}$ & Figure3 $\angle 15$ \\
\hline \multicolumn{4}{|l|}{ Continue 1} \\
\hline Indicators & Normal range & Evaluation method & Figure \\
\hline $\begin{array}{l}\text { Inclination of the } \\
\text { head }\end{array}$ & $88.8^{\circ} \sim 93.8^{\circ}$ & $\begin{array}{l}\text { Angle between glabella, menton, and a } \\
\text { horizontal line }\end{array}$ & Figure3 $\angle 17$ \\
\hline Footprint & $30 \% \sim 36 \%$ & $\begin{array}{l}\text { The ratio of the narrowest distance at the } \\
\text { inner and outer arches to the broadest } \\
\text { transverse distance at the forefoot }\end{array}$ & Figure3 \% \\
\hline $\begin{array}{l}\text { Difference in } \\
\text { length of both } \\
\text { lower limbs }\end{array}$ & $0.5 \mathrm{~cm}$ & $\begin{array}{l}\text { Measure with the length difference meter of } \\
\text { both lower limbs }\end{array}$ & \\
\hline $\begin{array}{l}\text { Note: LL:lower limb } \\
\text { female. }\end{array}$ & SIS=anterior $\mathrm{s}$ & erior iliac spine; PSIS=posterior superior iliac sp & M: man; F: \\
\hline
\end{tabular}

\section{Procedures}

Before preparing participants for global postural measurement on the GPS Lab, we determined bilateral leg length differences using the Delta Leg measuring device.

To make global postural indicator measurements using GPS Lab software, we placed marker stickers on specific bony landmarks on the participants' bodies. All of the participants were asked to undress to 
underwear, to expose as much skin as possible. The investigator palpated, confirmed, and placed markers on the spinous processes of $\mathrm{C} 7, \mathrm{~T} 3, \mathrm{~T} 6, \mathrm{~T} 9, \mathrm{~T} 12, \mathrm{~L} 3$, and S1, PSIS, the midpoint of the shoulder joint, the midpoint of the Achilles tendon, the midpoint of the lower third of the triceps surae, the anterior angle of the acromion, the midpoint of the acromion, the posterior angle of the acromion, the upper and lower angles of the scapula, the ASIS, the midpoint of the tibia, the lateral and medial condyles of the humerus, the larger trochanter of the femur, the midpoint of the lateral side of the knee joint, the lateral malleolus, the external canthus of the eye, and the tragus. The investigator checked the correct position of markers on bony landmarks through the cameras and software of the GPS Lab before photographing. The detailed procedures of the measurement are described in Supplementary 1 and a complete set of global posture photos of the subject are showed in Figure 4.

\section{Statistical analysis}

The MS Excel package was used to create a database and enter measurement values. Measurement data are presented as the mean and standard deviation. The comparison between groups was performed using the independent sample t-test. The Mann-Whitney $\mathrm{U}$ test was used if the values were not normally distributed. Categorical variables were described by the composition ratio and the chi-square test was used for comparison between groups. The odds ratio (OR) was calculated and the statistical significance level was defined as $p<0.05$.

\section{Results}

There was no significant difference in age (control: $22.52 \pm 1.93$ vs CNP: $23.45 \pm 1.91, p=0.070$ ), height (control: $1.65 \pm 0.08$ vs CNP: $1.65 \pm 0.08, p=0.779$ ), weight (control: $56.71 \pm 10.31$ vs CNP: $55.84 \pm 8.90$, $p=0.732$ ), and BMI (control: $20.63 \pm 2.47$ vs CNP: $20.49 \pm 2.03, p=0.806$ ) between the two groups (Table 2). The VAS score (control: $2.19 \pm 2.98$ vs CNP: $49.35 \pm 11.46, p<0.001$ ) and NDI (control: $1.60 \pm 2.38$ vs CNP: $27.55 \pm 9.07, p<0.001$ ) were significantly higher in the CNP group, while the posterior extensor muscle MIS (control: $18.25 \pm 4.50$ vs CNP: $11.50 \pm 2.43, p<0.001)$ and cervical flexion range (control: $75.48 \pm 10.04$ vs CNP: $65.32 \pm 15.00, p=0.004$ ) were significantly lower (Table 3 ).

Table 2

Basic conditions of the subjects (Mean \pm SD)

\begin{tabular}{|llllll|}
\hline Group & $\mathrm{n}(\mathrm{male} / \mathrm{female})$ & Age $(\mathrm{y})$ & Height $(\mathrm{m})$ & Weight $(\mathrm{kg})$ & $\mathrm{BMI}(\mathrm{kg} / \mathrm{m} 2)$ \\
\hline Control & $27(5 / 22)$ & $22.52 \pm 1.93$ & $1.65 \pm 0.08$ & $56.71 \pm 10.31$ & $20.63 \pm 2.47$ \\
\hline CNP & $31(6 / 25)$ & $23.45 \pm 1.91$ & $1.65 \pm 0.08$ & $55.84 \pm 8.90$ & $20.49 \pm 2.03$ \\
\hline $\mathbf{t}$ & & 1.846 & -0.282 & -0.345 & -0.247 \\
\hline $\mathrm{P}_{\text {value }}$ & & 0.070 & 0.779 & 0.732 & 0.806 \\
\hline
\end{tabular}


Table 3

Comparison of neck pain and disability indicators between the two subject groups (mean \pm SD)

\begin{tabular}{|c|c|c|c|c|c|}
\hline \multicolumn{2}{|l|}{ Indicator (unit) } & Control $(n=27)$ & CNP $(n=31)$ & $t$ & $p_{\text {value }}$ \\
\hline \multicolumn{2}{|l|}{ VAS score } & $2.19 \pm 2.98$ & $49.35 \pm 11.46$ & -22.083 & $<0.001$ ** \\
\hline \multicolumn{2}{|l|}{ NDI } & $1.60 \pm 2.38$ & $27.55 \pm 9.07$ & -15.331 & $<0.001^{\star *}$ \\
\hline \multicolumn{2}{|c|}{ Posterior extensor muscles MIS (kg) } & $18.25 \pm 4.50$ & $11.50 \pm 2.43$ & 6.959 & $<0.001^{\star *}$ \\
\hline \multirow[t]{6}{*}{ Cervical flexion activity $\left({ }^{\circ}\right)$} & Forward & $75.48 \pm 10.04$ & $65.32 \pm 15.00$ & 2.984 & 0.004 ** \\
\hline & Backward & $81.33 \pm 10.60$ & $74.61 \pm 15.92$ & 1.863 & 0.080 \\
\hline & Leftward & $49.41 \pm 8.11$ & $45.87 \pm 8.39$ & 1.627 & 0.109 \\
\hline & Rightward & $51.74 \pm 49.97$ & $49.97 \pm 12.14$ & 0.680 & 0.489 \\
\hline & Rotate left & $80.89 \pm 9.90$ & $73.81 \pm 21.96$ & 1.617 & 0.113 \\
\hline & Rotate right & $77.52 \pm 11.70$ & $73.10 \pm 21.02$ & 0.970 & 0.319 \\
\hline
\end{tabular}

In the lateral view, compared to the control group, the bilateral sagittal alignment of the body was significantly decreased in the CNP group (control [R/L]: $183.67 \pm 4.23 / 184.00 \pm 4.53$ vs CNP: $177.84 \pm$ $4.65 / 177.45 \pm 4.14, p<0.001 / p<0.001$ ). The left sagittal lower limb alignment (control: $1.30 \pm 4.18 \mathrm{vs}$ CNP: $4.23 \pm 5.82, p=0.004$ ) and the right cervical angle (control: $60.15 \pm 3.91$ vs CNP: $63.03 \pm 4.30, p=$ 0.010 ) were significantly increased in the CNP group. The other indicators were not statistically significant (Table 4). In the posterior view, only the right rear foot angle in the CNP group was significantly increased (control: $172.93 \pm 5.2$ vs CNP: $176.97 \pm 5.03, p=0.004$ ) compared to the control group, with no statistically significant differences in the other indicators (Table 5). The anterior view results (Table 6) showed that the anterior lower limb alignment of the CNP group was larger than that of the control group (control [R/L]: $184.26 \pm 2.63 / 184.41 \pm 3.03$ vs CNP: $178.71 \pm 3.23 / 178.52 \pm 3.31, p<0.001 / p<0.001$ ), as was the difference in the length of the lower limbs (control: $0.20 \pm 0.22$ vs CNP: $0.39 \pm 0.31, p=0.010$ ). However, there was no significant difference in other indicators between the two groups $(p>0.05)$. 
Table 4

Comparison of posture in the lateral view indicators between two groups

\begin{tabular}{|c|c|c|c|c|c|}
\hline Indicator (unit) & & Control(n=27) & CNP(n=31) & $t$ & $p_{\text {value }}$ \\
\hline \multirow[t]{2}{*}{ Vertical body position $\left({ }^{\circ}\right)$} & Right & $2.59 \pm 1.28$ & $2.13 \pm 1.61$ & 1.203 & 0.234 \\
\hline & Left & $1.96 \pm 1.09$ & $1.74 \pm 1.39$ & 0.666 & 0.58 \\
\hline \multirow[t]{2}{*}{ Sagittal alignment of the body $\left(\left(^{\circ}\right)\right.$} & Right & $183.67 \pm 4.23$ & $177.84 \pm 4.65$ & 4.963 & $<0.001^{\star \star}$ \\
\hline & Left & $184.00 \pm 4.53$ & $177.45 \pm 4.14$ & 5.751 & $<0.001^{\star \star}$ \\
\hline \multirow[t]{2}{*}{ Sagittal lower limbs alignment } & Right & $2.44 \pm 3.85$ & $3.68 \pm 5.69$ & -0.977 & 0.333 \\
\hline & Left & $1.30 \pm 4.18$ & $4.23 \pm 5.82$ & -2.221 & $0.031^{\star}$ \\
\hline \multirow[t]{2}{*}{ Inclination of the Pelvic $\left({ }^{\circ}\right)$} & Right & $14.93 \pm 6.38$ & $16.39 \pm 6.86$ & -0.834 & 0.407 \\
\hline & Left & $14.70 \pm 6.60$ & $16.13 \pm 6.37$ & -0.839 & 0.405 \\
\hline \multirow[t]{2}{*}{ Shoulder angle $\left(^{\circ}\right)$} & Right & $120.37 \pm 16.85$ & $123.77 \pm 18.93$ & -0.697 & 0.489 \\
\hline & Left & $118.52 \pm 18.93$ & $116.42 \pm 18.26$ & 0.429 & 0.669 \\
\hline \multirow[t]{2}{*}{ Cervical Angle } & Right & $60.15 \pm 3.91$ & $63.03 \pm 4.30$ & -2.657 & $0.010 *$ \\
\hline & Left & $60.30 \pm 3.69$ & $60.26 \pm 3.49$ & 0.041 & 0.968 \\
\hline \multirow[t]{2}{*}{ Sagittal head angle } & Right & $16.96 \pm 4.82$ & $15.42 \pm 3.28$ & 1.405 & 0.167 \\
\hline & Left & $16.07 \pm 4.71$ & $15.61 \pm 4.62$ & 0.376 & 0.708 \\
\hline
\end{tabular}

Table 5. Comparison of posture in the posterior view indicators between two groups 


\begin{tabular}{|c|c|c|c|c|c|}
\hline Indicator (unit) & & Control $\mid n=27 \square$ & $\mathrm{CNP}[\mathrm{n}=31)$ & $t$ & $\boldsymbol{p}_{\text {value }}$ \\
\hline \multirow[t]{2}{*}{ Rearfoot angle $\square^{\circ}[$} & Right & $172.93 \pm 5.22$ & $176.97 \pm 5.03$ & -3.000 & $0.004^{*}$ \\
\hline & Left & $175.70 \pm 5.67$ & $177.45 \pm 3.95$ & -1.376 & 0.174 \\
\hline Difference of bilateral PSIS height $\llbracket \mathrm{cm}[$ & & $-0.08 \pm 0.27$ & $-0.04 \pm 0.24$ & -0.640 & 0.525 \\
\hline $\begin{array}{l}\text { Distance of from bilateral PSIS to the } \\
\text { middle line } \llbracket \mathrm{cm} \square\end{array}$ & & $0.97 \pm 1.43$ & $1.17 \pm 1.40$ & -0.529 & 0.599 \\
\hline \multirow{2}{*}{$\begin{array}{l}\text { Alignment of the scapulas related to T3 } \\
\text { [cm }\end{array}$} & Right & $6.41 \pm 0.94$ & $6.65 \pm 0.96$ & -0.963 & 0.340 \\
\hline & Left & $5.88 \pm 0.93$ & $6.16 \pm 0.94$ & -1.144 & 0.258 \\
\hline $\begin{array}{l}\text { Difference in alignment of the scapulas } \\
\text { related to T3 on both sides } \square \mathrm{cm} \square\end{array}$ & & $0.53 \pm 0.77$ & $0.49 \pm 0.85$ & 0.184 & 0.855 \\
\hline Horizontal alignment of the $\left.\mathrm{PSISs} \square^{\circ}\right]$ & & $-0.33 \pm 1.41$ & $0.03 \pm 2.00$ & / & 0.847 \\
\hline \multirow[t]{2}{*}{ Scapular alignment $\square^{\circ} \square$} & Right & $0.04 \pm 5.89$ & $0.06 \pm 4.85$ & -0.019 & 0.985 \\
\hline & Left & $1.41 \pm 5.77$ & $1.74 \pm 4.65$ & -0.245 & 0.808 \\
\hline Horizontal alignment of the scapulas $\square^{\circ}[$ & & $-0.22 \pm 1.37$ & $0.26 \pm 1.48$ & -1.275 & 0.207 \\
\hline
\end{tabular}

Compared with the control group: * indicates $p<0.05$. PSIS=posterior superior iliac spine. 
Table 6

Comparison of posture in the anterior view indicators between two groups

\begin{tabular}{|c|c|c|c|c|c|}
\hline Indicator (unit) & & Control(n=27) & CNP(n=31) & $t$ & $p_{\text {value }}$ \\
\hline \multirow[t]{2}{*}{ Anterior lower limbs alignment } & Right & $184.26 \pm 2.63$ & $178.71 \pm 3.23$ & 7.116 & $<0.001^{\star *}$ \\
\hline & Left & $184.41 \pm 3.03$ & $178.52 \pm 3.31$ & 7.038 & $<0.001^{\star *}$ \\
\hline \multirow[t]{2}{*}{$Q$ angle(M=5:6) } & Right & $12.80 \pm 6.98$ & $20.17 \pm 14.16$ & -1.055 & 0.319 \\
\hline & Left & $11.20 \pm 5.40$ & $14.17 \pm 7.57$ & -0.732 & 0.483 \\
\hline \multirow[t]{2}{*}{$\mathrm{Q}$ angle(F=22:25) } & Right & $25.236 \pm 8.86$ & $22.30 \pm 7.46$ & 1.2 & 0.237 \\
\hline & Left & $23.23 \pm 7.93$ & $21.30 \pm 9.13$ & 0.753 & 0.456 \\
\hline $\begin{array}{l}\text { Distance from bilateral ASIS to the } \\
\text { middle line }(\mathrm{cm})\end{array}$ & & $-0.24 \pm 1.17$ & $-0.42 \pm 1.51$ & 0.489 & 0.627 \\
\hline Bilateral ASIS height difference(cm) & & $0.02 \pm 0.57$ & $-0.03 \pm 0.72$ & 0.256 & 0.799 \\
\hline Horizontal alignment of the ASISs $\left({ }^{\circ}\right)$ & & $0.19 \pm 1.69$ & $0.03 \pm 2.04$ & 0.308 & 0.759 \\
\hline $\begin{array}{l}\text { Horizontal alignment of the } \\
\text { acromions }\left(^{(}\right)\end{array}$ & & $-0.89 \pm 1.19$ & $-0.39 \pm 1.41$ & / & 0.721 \\
\hline Horizontal alignment of head & & $-0.15 \pm 1.73$ & $-0.35 \pm 2.14$ & 0.401 & 0.69 \\
\hline Inclination of the head & & $90.22 \pm 1.58$ & $90.49 \pm 1.43$ & -0.662 & 0.511 \\
\hline \multirow[t]{2}{*}{ Footprint(\%) } & Right & $28.22 \pm 10.69$ & $30.23 \pm 7.78$ & -0.823 & 0.414 \\
\hline & Left & $28.04 \pm 12.10$ & $29.90 \pm 9.14$ & -0.663 & 0.51 \\
\hline $\begin{array}{l}\text { Difference in length of the lower } \\
\text { limbs(cm) }\end{array}$ & & $0.20 \pm 0.22$ & $0.39 \pm 0.31$ & -2.67 & $0.010^{\star}$ \\
\hline $\begin{array}{l}\text { Compared with the control group: * ir } \\
\text { spine. }\end{array}$ & tho & 0.05. M: Male, $F$ & Female. $A S I S=$ & & erior iliac \\
\hline
\end{tabular}

We also investigated whether any abnormal indicators could be attributed to CNP. By calculating the OR, the results showed that the right foot valgus and the sagittal alignment of the body were smaller, the sagittal lower limb alignment and the anterior lower limb alignment were greater than the normal range, and the OR value for neck pain was $6.667,6.061,7.125,0.034$, respectively. However, there was no statistical significance in other indicators (Table 7). 
Table 7

Analysis of variables and OR calculations of single factor $\chi^{2}$ with or without CNP

\begin{tabular}{|c|c|c|c|c|c|c|c|}
\hline \multirow[t]{2}{*}{ Variable } & \multirow[t]{2}{*}{ Groups } & \multirow[t]{2}{*}{ Yes } & \multirow[t]{2}{*}{ No } & \multirow[t]{2}{*}{$x^{2}$} & \multirow[t]{2}{*}{ OR } & \multicolumn{2}{|c|}{ OR value $95 \% \mathrm{Cl}$} \\
\hline & & & & & & $\begin{array}{l}\text { Lower } \\
\text { limit }\end{array}$ & $\begin{array}{l}\text { Upper } \\
\text { limit }\end{array}$ \\
\hline \multirow{2}{*}{$\begin{array}{l}\text { Difference in length of } \\
\text { the both lower limbs }\end{array}$} & greater & 6 & 4 & 0.012 & 1.38 & 0.345 & 5.519 \\
\hline & normal & 25 & 23 & & & & \\
\hline \multirow{2}{*}{$\begin{array}{l}\text { Vertical body position } \\
\text { (right) }\end{array}$} & greater & 7 & 7 & 0.096 & 0.826 & 0.246 & 2.773 \\
\hline & normal & 23 & 19 & & & & \\
\hline \multirow{2}{*}{$\begin{array}{l}\text { Vertical body position } \\
\text { (right) }\end{array}$} & less & 1 & 1 & 0 & \#\# & \#\# & \#\# \\
\hline & normal & 23 & 19 & & & & \\
\hline \multirow{2}{*}{$\begin{array}{l}\text { Vertical body position } \\
\text { (left) }\end{array}$} & less & 3 & 3 & 0 & \#\# & \#\# & \#\# \\
\hline & normal & 28 & 23 & & & & \\
\hline \multirow{2}{*}{$\begin{array}{l}\text { Vertical body position } \\
\text { (left) }\end{array}$} & less & 0 & 1 & 0.006 & 1.043 & 0.96 & 1.134 \\
\hline & normal & 28 & 23 & & & & \\
\hline \multirow{2}{*}{$\begin{array}{l}\text { Sagittal alignment of the } \\
\text { body (right) }\end{array}$} & less & 25 & 11 & $9.760 \star \star$ & 6.061 & 1.87 & 19.647 \\
\hline & normal & 6 & 16 & & & & \\
\hline \multirow{2}{*}{$\begin{array}{l}\text { Sagittal alignment of the } \\
\text { body (right) }\end{array}$} & greater & 0 & 0 & \#\# & $\# \#$ & $\# \#$ & \#\# \\
\hline & normal & 6 & 16 & & & & \\
\hline \multirow{2}{*}{$\begin{array}{l}\text { Sagittal alignment of the } \\
\text { body (left) }\end{array}$} & less & 28 & 10 & $17.114^{\star \star}$ & 14.933 & 3.577 & 62.337 \\
\hline & normal & 3 & 16 & & & & \\
\hline \multirow{2}{*}{$\begin{array}{l}\text { Sagittal alignment of the } \\
\text { body (left) }\end{array}$} & greater & 0 & 1 & 0 & \#\# & \#\# & \#\# \\
\hline & normal & 3 & 16 & & & & \\
\hline \multirow[t]{2}{*}{ Arch status(right) } & talipes cavus & 13 & 15 & 0.861 & 0.578 & 0.181 & 1.848 \\
\hline & normal & 12 & 8 & & & & \\
\hline \multirow[t]{2}{*}{ Arch status(right) } & Low arches & 6 & 4 & 0 & 1 & 0.212 & 4.709 \\
\hline & normal & 12 & 8 & & & & \\
\hline Arch status(left) & talipes cavus & 15 & 13 & 0.017 & 0.923 & 0.281 & 3.034 \\
\hline
\end{tabular}




\begin{tabular}{|c|c|c|c|c|c|c|c|}
\hline \multirow[t]{2}{*}{ Variable } & \multirow[t]{2}{*}{ Groups } & \multirow[t]{2}{*}{ Yes } & \multirow[t]{2}{*}{ No } & \multirow[t]{2}{*}{$x^{2}$} & \multirow[t]{2}{*}{ OR } & \multicolumn{2}{|c|}{ OR value $95 \% \mathrm{Cl}$} \\
\hline & & & & & & $\begin{array}{l}\text { Lower } \\
\text { limit }\end{array}$ & $\begin{array}{l}\text { Upper } \\
\text { limit }\end{array}$ \\
\hline & normal & 10 & 8 & & & & \\
\hline \multirow[t]{2}{*}{ Arch status(left) } & Low arches & 6 & 6 & 0.089 & 0.8 & 0.185 & 3.46 \\
\hline & normal & 10 & 8 & & & & \\
\hline \multirow[t]{2}{*}{ Rear foot angle (right) } & strephenopodia & 3 & 7 & 0.072 & 0.612 & 0.135 & 2.786 \\
\hline & normal & 14 & 20 & & & & \\
\hline \multirow[t]{2}{*}{ Rear foot angle (right) } & strephexopodia & 14 & 3 & 7.761 *夫 & 6.667 & 1.609 & 27.629 \\
\hline & normal & 14 & 20 & & & & \\
\hline \multirow[t]{2}{*}{ Rear foot angle (left) } & strephenopodia & 2 & 4 & 0.262 & 0.417 & 0.067 & 2.599 \\
\hline & normal & 18 & 15 & & & & \\
\hline \multirow[t]{2}{*}{ Rear foot angle (left) } & strephenopodia & 11 & 8 & 0.055 & 1.146 & 0.367 & 3.582 \\
\hline & normal & 18 & 5 & & & & \\
\hline \multirow{2}{*}{$\begin{array}{l}\text { Sagittal lower limbs } \\
\text { alignment (right) }\end{array}$} & Knee flexion & 15 & 5 & $7.215^{\star \star}$ & 5.4 & 1.511 & 19.297 \\
\hline & normal & 10 & 18 & & & & \\
\hline \multirow[t]{2}{*}{$\begin{array}{l}\text { Sagittal lower limbs } \\
\text { alignment (right) }\end{array}$} & $\begin{array}{l}\text { Knee } \\
\text { overextension }\end{array}$ & 6 & 4 & 0.926 & 2.7 & 0.613 & 11.892 \\
\hline & normal & 10 & 8 & & & & \\
\hline \multirow{2}{*}{$\begin{array}{l}\text { Sagittal lower limbs } \\
\text { alignment (left) }\end{array}$} & Knee flexion & 17 & 2 & $15.322^{\star \star}$ & 19.125 & 3.545 & 103.163 \\
\hline & normal & 8 & 18 & & & & \\
\hline \multirow[t]{2}{*}{$\begin{array}{l}\text { Sagittal lower limbs } \\
\text { alignment (left) }\end{array}$} & $\begin{array}{l}\text { Knee } \\
\text { overextension }\end{array}$ & 6 & 8 & 0.891 & 1.929 & 0.489 & 7.605 \\
\hline & normal & 8 & 18 & & & & \\
\hline \multicolumn{8}{|l|}{ Continue 1} \\
\hline \multirow[t]{2}{*}{ Variable } & Groups & Yes & No & $x^{2}$ & OR & \multicolumn{2}{|c|}{ OR value $95 \% \mathrm{Cl}$} \\
\hline & & & & & & $\begin{array}{l}\text { Lower } \\
\text { limit }\end{array}$ & $\begin{array}{l}\text { Upper } \\
\text { limit }\end{array}$ \\
\hline $\begin{array}{l}\text { Greater than indicates } \\
\text { range; right high indica } \\
\text { \#\# indicates that the s } \\
\text { indicates } p<0.05 \text {, and }\end{array}$ & $\begin{array}{l}\text { cannot calculate } \\
\text { licates } p<0.01\end{array}$ & excee & & $\begin{array}{l}\text { s than indi } \\
\text { high indice } \\
\text { ata has a }\end{array}$ & $\begin{array}{l}\text { tes lowe } \\
\text { es that th } \\
\text { 'value ol }\end{array}$ & $\begin{array}{l}\text { han the } \\
\text { left side } \\
\text { too sma }\end{array}$ & $\begin{array}{l}\text { ormal } \\
\text { s high; } \\
\text { ll. * }\end{array}$ \\
\hline
\end{tabular}




\begin{tabular}{|c|c|c|c|c|c|c|c|}
\hline \multirow[t]{2}{*}{ Variable } & \multirow[t]{2}{*}{ Groups } & \multirow[t]{2}{*}{ Yes } & \multirow[t]{2}{*}{ No } & \multirow[t]{2}{*}{$x^{2}$} & \multirow[t]{2}{*}{ OR } & \multicolumn{2}{|c|}{ OR value $95 \% \mathrm{C}$} \\
\hline & & & & & & $\begin{array}{l}\text { Lower } \\
\text { limit }\end{array}$ & $\begin{array}{l}\text { Upper } \\
\text { limit }\end{array}$ \\
\hline \multirow{2}{*}{$\begin{array}{l}\text { Anterior lower limbs } \\
\text { alignment (right) }\end{array}$} & gonyectyposis & 2 & 20 & $26.454^{\star \star}$ & 0.026 & 0.005 & 0.138 \\
\hline & normal & 27 & 7 & & & & \\
\hline \multirow{2}{*}{$\begin{array}{l}\text { Anterior lower limbs } \\
\text { alignment (right) }\end{array}$} & genu valgum & 2 & 0 & 0 & $\# \#$ & \# \# & $\# \#$ \\
\hline & normal & 27 & 7 & & & & \\
\hline \multirow{2}{*}{$\begin{array}{l}\text { Anterior lower limbs } \\
\text { alignment (left) }\end{array}$} & gonyectyposis & 2 & 19 & $22.519 \star \star$ & & 0.034 & 0.006 \\
\hline & normal & 25 & 8 & & & & \\
\hline \multirow{2}{*}{$\begin{array}{l}\text { Anterior lower limbs } \\
\text { alignment (left) }\end{array}$} & genu valgum & 4 & 0 & 0.22 & $\# \#$ & $\# \#$ & \#\# \\
\hline & normal & 25 & 8 & & & & \\
\hline \multirow[t]{2}{*}{ Quadriceps angle (right) } & increase & 19 & 16 & 0.461 & 1.484 & 0.473 & 4.656 \\
\hline & normal & 8 & 10 & & & & \\
\hline \multirow[t]{2}{*}{ Quadriceps angle (right) } & decrease & 4 & 1 & 0.814 & 5 & 0.463 & 54.044 \\
\hline & normal & 8 & 10 & & & & \\
\hline \multirow[t]{2}{*}{ Quadriceps angle (left) } & increase & 19 & 16 & 0.1 & 1.188 & 0.408 & 3.455 \\
\hline & normal & 11 & 11 & & & & \\
\hline \multirow[t]{2}{*}{ Quadriceps angle (left) } & decrease & 1 & 0 & 0 & $\# \#$ & $\# \#$ & \#\# \\
\hline & normal & 11 & 11 & & & & \\
\hline \multirow{2}{*}{$\begin{array}{l}\text { Inclination of Pelvic } \\
\text { (right) }\end{array}$} & Lean forward & 13 & 8 & 0.93 & 1.727 & 0.567 & 5.262 \\
\hline & normal & 16 & 17 & & & & \\
\hline \multirow{2}{*}{$\begin{array}{l}\text { Inclination of Pelvic } \\
\text { (right) }\end{array}$} & Lean back & 2 & 2 & 0 & 1.063 & 0.133 & 8.466 \\
\hline & normal & 16 & 17 & & & & \\
\hline \multirow[t]{2}{*}{ Inclination of Pelvic (left) } & Lean forward & 11 & 9 & 0.026 & 1.094 & 0.365 & 3.277 \\
\hline & normal & 19 & 17 & & & & \\
\hline \multirow[t]{2}{*}{ Inclination of Pelvic (left) } & Lean back & 1 & 1 & 0 & 0.895 & 0.052 & 15.437 \\
\hline & normal & 19 & 17 & & & & \\
\hline $\begin{array}{l}\text { Greater than indicates tha } \\
\text { range; right high indicates } \\
\text { \#\# indicates that the syst } \\
\text { indicates } p<0.05 \text {, and } * *\end{array}$ & $\begin{array}{l}\text { cannot calculat } \\
\text { icates } p<0.01 \text {. }\end{array}$ & xces & ed, I & s than inc & $\begin{array}{l}\text { tes lov } \\
\text { es that } \\
\text { "value }\end{array}$ & than the & $\begin{array}{l}\text { ormal } \\
\text { s high; } \\
\text { ll. * }\end{array}$ \\
\hline
\end{tabular}




\begin{tabular}{|c|c|c|c|c|c|c|c|}
\hline \multirow[t]{2}{*}{ Variable } & \multirow[t]{2}{*}{ Groups } & \multirow[t]{2}{*}{ Yes } & \multirow[t]{2}{*}{ No } & \multirow[t]{2}{*}{$x^{2}$} & \multirow[t]{2}{*}{ OR } & \multicolumn{2}{|c|}{ OR value $95 \% \mathrm{Cl}$} \\
\hline & & & & & & $\begin{array}{l}\text { Lower } \\
\text { limit }\end{array}$ & $\begin{array}{l}\text { Upper } \\
\text { limit }\end{array}$ \\
\hline \multirow[t]{2}{*}{ Pelvic state } & abnormal & 17 & 13 & 0.259 & 1.308 & 0.465 & 3.68 \\
\hline & normal & 14 & 14 & & & & \\
\hline \multirow{2}{*}{$\begin{array}{l}\text { Horizontal alignment of } \\
\text { the ASISs }\end{array}$} & right high & 3 & 2 & 0 & 1.5 & 0.227 & 9.916 \\
\hline & normal & 21 & 21 & & & & \\
\hline \multirow{2}{*}{$\begin{array}{l}\text { Horizontal alignment of } \\
\text { the ASISs }\end{array}$} & left high & 7 & 4 & 0.65 & 1.75 & 0.445 & 6.882 \\
\hline & normal & 21 & 21 & & & & \\
\hline \multirow{2}{*}{$\begin{array}{l}\text { Horizontal alignment of } \\
\text { the PSISs }\end{array}$} & right high & 2 & 0 & 0.279 & \#\# & \#\# & \#\# \\
\hline & normal & 27 & 22 & & & & \\
\hline \multirow{2}{*}{$\begin{array}{l}\text { Horizontal alignment of } \\
\text { the PSISs }\end{array}$} & left high & 2 & 5 & 0.828 & 0.326 & 0.058 & 1.845 \\
\hline & normal & 27 & 22 & & & & \\
\hline \multirow[t]{2}{*}{ Shoulder angle (right) } & less & 15 & 14 & 0.082 & 1.179 & 0.383 & 3.627 \\
\hline & normal & 10 & 11 & & & & \\
\hline \multirow[t]{2}{*}{ Shoulder angle (right) } & greater & 6 & 2 & 1.736 & 4.5 & 0.752 & 26.931 \\
\hline & normal & 10 & 11 & & & & \\
\hline \multirow[t]{2}{*}{ Shoulder angle (left) } & less & 17 & 16 & 0.163 & 0.797 & 0.265 & 2.397 \\
\hline & normal & 12 & 9 & & & & \\
\hline \multirow[t]{2}{*}{ Shoulder angle (left) } & greater & 2 & 2 & 0 & 0.75 & 0.088 & 6.388 \\
\hline & normal & 12 & 9 & & & & \\
\hline \multirow{2}{*}{$\begin{array}{l}\text { Difference in Alignment } \\
\text { of the scapulas related to } \\
\text { T3 }\end{array}$} & abnormal & 4 & 5 & 0.051 & 1.534 & 0.367 & 6.412 \\
\hline & normal & 27 & 22 & & & & \\
\hline \multicolumn{8}{|l|}{ Continue 2} \\
\hline \multirow[t]{2}{*}{ Variable } & Groups & Yes & No & $x^{2}$ & OR & OR valu & $95 \% \mathrm{Cl}$ \\
\hline & & & & & & $\begin{array}{l}\text { Lower } \\
\text { limit }\end{array}$ & $\begin{array}{l}\text { Upper } \\
\text { limit }\end{array}$ \\
\hline $\begin{array}{l}\text { Greater than indicates that } \\
\text { range; right high indicates } \\
\text { \#\# indicates that the syste } \\
\text { indicates } p<0.05 \text {, and } * *\end{array}$ & $\begin{array}{l}\text { cannot cal } \\
\text { licates } p<0\end{array}$ & excee & & $\begin{array}{l}\text { s than it } \\
\text { high inc } \\
\text { ata has }\end{array}$ & tes lon & $\begin{array}{l}\text { han the } \\
\text { left side } \\
\text { too sm }\end{array}$ & $\begin{array}{l}\text { ormal } \\
\text { s high; } \\
\text { ll. * }\end{array}$ \\
\hline
\end{tabular}




\begin{tabular}{|c|c|c|c|c|c|c|c|}
\hline \multirow[t]{2}{*}{ Variable } & \multirow[t]{2}{*}{ Groups } & \multirow[t]{2}{*}{ Yes } & \multirow[t]{2}{*}{ No } & \multirow[t]{2}{*}{$x^{2}$} & \multirow[t]{2}{*}{ OR } & \multicolumn{2}{|c|}{ OR value $95 \% \mathrm{Cl}$} \\
\hline & & & & & & $\begin{array}{l}\text { Lower } \\
\text { limit }\end{array}$ & $\begin{array}{l}\text { Upper } \\
\text { limit }\end{array}$ \\
\hline \multirow[t]{2}{*}{$\begin{array}{l}\text { Scapular alignment } \\
\text { (right) }\end{array}$} & $\begin{array}{l}\text { Downward } \\
\text { swing }\end{array}$ & 18 & 13 & 0.206 & 1.278 & 0.443 & 3.691 \\
\hline & normal & 13 & 12 & & & & \\
\hline \multirow{2}{*}{$\begin{array}{l}\text { Scapular alignment } \\
\text { (right) }\end{array}$} & upward swing & 0 & 2 & 0.464 & \#\# & $\# \#$ & \#\# \\
\hline & normal & 13 & 12 & & & & \\
\hline \multirow[t]{2}{*}{ Scapular alignment (left) } & $\begin{array}{l}\text { Downward } \\
\text { swing }\end{array}$ & 14 & 12 & 0.006 & 0.961 & 0.337 & 2.736 \\
\hline & normal & 17 & 14 & & & & \\
\hline \multirow[t]{2}{*}{ Scapular alignment (left) } & upward swing & 0 & 1 & 0.004 & \#\# & \#\# & \#\# \\
\hline & normal & 17 & 14 & & & & \\
\hline \multirow{2}{*}{$\begin{array}{l}\text { Horizontal alignment of } \\
\text { the scapula }\end{array}$} & abnormal & 0 & 0 & \#\# & \#\# & $\# \#$ & \#\# \\
\hline & normal & 31 & 27 & & & & \\
\hline \multirow{2}{*}{$\begin{array}{l}\text { Horizontal alignment of } \\
\text { the acromions }\end{array}$} & left high & 7 & 3 & 0.648 & 2.333 & 0.539 & 10.109 \\
\hline & normal & 24 & 24 & & & & \\
\hline \multirow[t]{2}{*}{ Cervical angle(right) } & greater & 30 & 23 & 1.209 & 0.192 & 0.02 & 1.832 \\
\hline & normal & 1 & 4 & & & & \\
\hline \multirow[t]{2}{*}{ Cervical angle(left) } & greater & 23 & & 0 & 1.106 & 0.265 & 4.618 \\
\hline & normal & 4 & & & & & \\
\hline \multirow{2}{*}{$\begin{array}{l}\text { Sagittal head angle } \\
\text { (right) }\end{array}$} & greater & 2 & & 0.015 & 0.417 & 0.036 & 4.869 \\
\hline & normal & 25 & & & & & \\
\hline \multirow[t]{2}{*}{ Sagittal head angle (left) } & greater & 24 & & 0.251 & 1.92 & 0.431 & 8.56 \\
\hline & normal & 3 & & & & & \\
\hline \multirow{2}{*}{$\begin{array}{l}\text { Horizontal alignment of } \\
\text { head }\end{array}$} & right high & 0 & & 0 & \#\# & \#\# & \#\# \\
\hline & normal & 21 & & & & & \\
\hline $\begin{array}{l}\text { Horizontal alignment of } \\
\text { head }\end{array}$ & left high & 6 & & 0.151 & 1.273 & 0.377 & 4.293 \\
\hline \multicolumn{8}{|c|}{$\begin{array}{l}\text { Greater than indicates that the normal range is exceeded, less than indicates lower than the normal } \\
\text { range; right high indicates that the right side is high, and left high indicates that the left side is high; } \\
\text { \#\# indicates that the system cannot calculate because the data has a " } 0 \text { " value or is too small. }{ }^{*} \\
\text { indicates } p<0.05 \text {, and }{ }^{* *} \text { indicates } p<0.01 \text {. }\end{array}$} \\
\hline
\end{tabular}




\begin{tabular}{|c|c|c|c|c|c|c|c|}
\hline \multirow[t]{2}{*}{ Variable } & \multirow[t]{2}{*}{ Groups } & \multirow[t]{2}{*}{ Yes } & \multirow[t]{2}{*}{ No } & \multirow[t]{2}{*}{$x^{2}$} & \multirow[t]{2}{*}{ OR } & \multicolumn{2}{|c|}{ OR value $95 \% \mathrm{Cl}$} \\
\hline & & & & & & $\begin{array}{l}\text { Lower } \\
\text { limit }\end{array}$ & $\begin{array}{l}\text { Upper } \\
\text { limit }\end{array}$ \\
\hline & normal & 21 & & & & & \\
\hline \multirow[t]{2}{*}{ Inclination of the head } & Left leaning & 4 & & 0 & 0.852 & 0.191 & 3.792 \\
\hline & normal & \multicolumn{6}{|l|}{23} \\
\hline \multicolumn{8}{|c|}{$\begin{array}{l}\text { Greater than indicates that the normal range is exceeded, less than indicates lower than the normal } \\
\text { range; right high indicates that the right side is high, and left high indicates that the left side is high; } \\
\text { \#\# indicates that the system cannot calculate because the data has a " } 0 \text { " value or is too small. }{ }^{*} \\
\text { indicates } p<0.05 \text {, and ** indicates } p<0.01 \text {. }\end{array}$} \\
\hline
\end{tabular}

\section{Discussion}

The results of the current study showed no statistically significant differences in postural indicators other than cervical angle. A prior study by Yip et al. ${ }^{[19]}$ found that patients with CNP hold their head more extended than healthy individuals, and, compared to a healthy population, the sagittal cervical angle is increased, suggesting that the posterior extensor muscles are tense or shortened while the cervical flexors are in a passively elongated state. An increase in the cervical angle may therefore be one of the risk factors for the occurrence and development of craniofacial pain, headache, neck and shoulder pain, decreased joint mobility, and muscle stiffness and tenderness ${ }^{[22]}$. However, the study by Ghamkhar et al. [20] found that forward head posture was not related to neck pain. Another prospective cohort study revealed that a forward head in sitting posture in late adolescence was associated with a reduced risk of persistent neck pain in young adults ${ }^{[23]}$. This difference may be due to the measurement method. It is worth noting that as the posture of the body changes gradually throughout life, tissue plasticity and adaptability may also contribute to a linear relationship between symptoms and structural changes.

We also showed that the sagittal alignment of the body in students with CNP is significantly lower than that in the healthy control group, meaning that the trunk of students with CNP leans more forward than the hips. The forward tilt of the trunk causes the body's center of gravity to move forward, passively elongating the spine's extensor muscles for longer periods. From the perspective of a mechanical lever, the body must increase the corresponding resistance as the torque becomes higher. To balance dynamic torque, the spine extensor muscles, such as the erector spinae and other lumbar muscles posterior to the spine, must generate greater tension, resulting in them becoming overloaded over time. Our results showed that the mean angle of the left sagittal lower limb alignment in patients with CNP was larger than that in the control group and that the left knee joint in students with CNP showed obvious knee flexion during quiet, normal standing. This suggests that the patient's knee flexor and extensor muscles have a lower ratio or that the hamstring and calf triceps are tense and shortened, and the quadriceps muscle is elongated and weakened in patients with CNP ${ }^{[16,24]}$. The results of the chi-square test and OR suggests that the decrease of the bilateral sagittal alignment of the body and the increase of the bilateral sagittal 
lower limb alignment are the risk factors for CNP. Conversely, our results showed that differences in shoulder angle between the two groups were not significant, suggesting that the shoulder angle does not contribute to an increased risk of developing CNP.

From the global perspective of anatomical trains or muscle chains, postural balance in the sagittal plane is mainly controlled by a tension-relaxation relationship between the superficial front lines (SFL) and superficial back lines (SBL) ${ }^{[16]}$. Dysfunction of the SFL can cause the body to lean forward or limit the ability of the body to extend backward. Our study found that patients with CNP leaned their body forward more, increasing the sagittal cervical spine angle, indicating that the SFL is in a shortened and tensed state, while the SBL is elongated. To prevent the trunk from falling forwards, the muscles in the SBL continue to contract and tension increases. Under this "wave effect" in the kinematic chains, abnormal muscle tension and pressure are transmitted caudally and may result in abnormal muscle tension and tightness in the hamstring and gastrocnemius muscles. The cervical vertebrae are the weakest part of the entire spine structure and are more prone to morphological changes in the process of power transmission. The deep front line (DFL) also maintains balance in the trunk and neck. Studies have shown that the muscle strength of the posterior cervical extensor muscles and the deep flexor muscles of patients with CNP is lower than those without neck pain [24, 25]; this may explain the knee flexion observation in patients with CNP in our study.

We also measured several postural indicators in the frontal plane. The results showed that the rear foot angle and the difference in the length of the lower limbs in the CNP group were larger than those in the control group, while the bilateral anterior lower limb alignments were smaller than the control group. The rear foot angle is an important reference for evaluating ankle valgus. From a global perspective, the cause of this difference may be related to the spiral line (SPL) in the myofascial lines. The SPL functions posturally to wrap the body in a double spiral, which helps to maintain balance across all planes, connects the foot arches with the pelvic angle, and helps to determine efficient knee-tracking in walking. In a state of imbalance, the SPL participates in creating, compensating for, and maintaining twists, rotations, and lateral shifts in the body ${ }^{[16]}$. We found that the abnormal link in the posture of students with CNP overlaps with the SPL, including the splenius capitis and cervicis, tibialis anterior, peroneus brevis, and peroneus longus. If we consider the suboccipital muscles that connect to the occipital and the long bones of the tibia that connect the foot bones to be the head and tail of the train, respectively, when the mechanics change in the front of the head, the tail will also change accordingly. This mechanical transmission may explain the occurrence of foot valgus in patients with CNP.

Studies have also shown that knee varus is associated with a decrease in the rearfoot angle and that this can cause adverse effects in the frontal plane of the ipsilateral lower extremity [26]. Our results showed that the knee varus angle of the control subjects was smaller than that of the CNP subjects, which is inconsistent with the results of the aforementioned study. In our study, the sagittal lower limb alignment of students with CNP was smaller than that of the control group, that is, the knee joint was not fully extended. According to the movement characteristics of the knee joint, the force area of the inner side of the knee joint is larger than that of the outer side when fully extended, the tibia is rolled and in torsion 
relative to the femur, and the knee varus angle is increased at this time ${ }^{[27,28]}$. We also found that the length difference of the lower limbs in students with CNP was significantly higher than that in the control group, which may also have caused the flexion angle of the longer leg's knee joint to be larger than the shorter side when the pressure on the two feet is the same. Another possible mechanism is adaptive neuromuscular activity compensation in patients with CNP, but more relevant mechanism studies and larger sample studies are needed to confirm this.

The OR is an indicator of the power of the association between disease and exposure. We demonstrate that the OR values of whether the angle of the sagittal body alignment on both sides was smaller than the normal range, whether the right foot was valgus, whether the angle between the left and right sagittal lower limbs was greater than the normal range, and whether there was neck pain or not, were 6.061, 6.667 , and 7.125 , respectively, indicating that subjects with trunk flexion and rearfoot valgus were more likely to present with CNP. In subjects with normal rearfoot angle, subjects with knee flexion were more likely to have neck pain than subjects with normal knee sagittal posture. The OR value of whether the anterior lower limb alignment is greater than the normal range and the neck pain is $<0.034$, indicating that knee varus is not a risk factor for neck pain.

\section{Limitations}

This study has several limitations that warrant discussion. First. we measured the overall posture but did not conduct in-depth research on the physiological state of the muscles, joints, and other structures (such as muscle strength, hardness, and joint activity), and we did not apply these theories to practice. Therefore, it is necessary to expand the sample size and include more people in different occupations in future research.

\section{Conclusions}

In conclusion, compared to healthy people without neck pain, students with CNP have a larger difference in the length of both lower extremities, a smaller ankle varus angle, a larger knee flexion angle, a forward tilt of the pelvis, and an increased extension of the head and neck on the right side. Leaning forward, foot valgus, and knee flexion on both sides are risk factors for CNP. Our results indicate that patients with CNP have a more deviant posture in the sagittal plane than healthy people without neck pain, suggesting that restoring the balance of the SBL and SFL will help to treat CNP. From a global postural perspective, strengthening weak muscle groups in the DFL and SPL may improve any dysfunctions in these lines. Meanwhile, tense, shortened muscle groups should be released and lengthened or stretched to improve abnormal posture and restore the mechanical balance to treat patients with CNP and prevent sequelae.

\section{Abbreviations}

ASIS: anterior superior iliac spine; CNP: chronic neck pain; FHP: forward head posture; GPS: global postural systems; MIS: maximums isometric strength; NDI: neck disability index; OR: odds ratio; PSIS: 
posterior superior iliac spine; SBL: superficial back lines; SFL: superficial front lines; SPL: spiral line; VAS: visual analogue scale.

\section{Declarations}

\section{Ethics approval and consent to participate}

The study was approved by the Guangzhou Sport University (IRB approval number: 2018LCLL-12). All methods of this study were performed in accordance with the Declaration of Helsinki. The informed consent document was seen as Supplementary 1.

\section{Consent for publication}

We also have obtained the consent from the subject who agreed to publish the images in this paper and signed the informed consent (Supplementary 2).

\section{Availability of data and materials}

The datasets used and/or analyzed during the current study are available from the corresponding author on reasonable request.

\section{Competing interests}

All of the authors declare no competing interests.

\section{Funding}

This research did not receive any specific grant from funding agencies in the public, commercial, or notfor-profit sectors.

\section{Authors' contributions}

The study was designed by J.G.Z. and W.H.W., L.G.Y. contributed to data collection and X.H.H supervised the experiment processing and data analyses. J.G.Z. and W.H.W. wrote the manuscript.

\section{Acknowledgements}

We would like to thank Zhenzhu Zhang, Hao Lan and Zhijian Zou provided great help during the implementation of this experiment, including pasting surface markers to the subjects, debugging equipment, etc. Zhi Zou, Bixiao Song and Liru Liu provided valuable suggestions and modifications during the writing of the article. We also thank LetPub (www.letpub.com) for its linguistic assistance during the preparation of this manuscript.

\section{Author details}


${ }^{1}$ School of Sport and Health, Guangzhou Sport University, Guangzhou 510500, Guangdong province, China; ${ }^{2}$ College of Kinesiology, Shanghai University of Sport, Shanghai 200438, China; ${ }^{3}$ Guangzhou College of Applied Science and Technology, Guangzhou, Guangdong province, China.

\section{References}

1. Safiri S, Kolahi A A, Hoy D, Buchbinder R, Mansournia M A, Bettampadi D, Ashrafi-Asgarabad A, et al., Global, regional, and national burden of neck pain in the general population, 1990-2017: systematic analysis of the Global Burden of Disease Study 2017. BMJ, 2020, 368:m791.

2. Jin Z, Wang D, Zhang H, Liang J, Feng X, Zhao J,Sun L, Incidence trend of five common musculoskeletal disorders from 1990 to 2017 at the global, regional and national level: results from the global burden of disease study 2017. Ann Rheum Dis, 2020, 79(8):1014-1022.

3. Daher A, Carel R S, Tzipi K, Esther H,Dar G, The effectiveness of an aerobic exercise training on patients with neck pain during a short- and long-term follow-up: a prospective double-blind randomized controlled trial. Clin Rehabil, 2020, 34(5):617-629.

4. Yamato T P, Saragiotto B T,Maher C, Therapeutic exercise for chronic non-specific neck pain: PEDro systematic review update. Br J Sports Med, 2015, 49(20):1350.

5. Lascurain-Aguirrebena I, Newham D J, Casado-Zumeta X, Lertxundi A,Critchley D J, Immediate effects of cervical mobilisations on neck muscle activity during active neck movements in patients with non-specific neck pain. A double blind placebo controlled trial. Physiotherapy, 2021, 110:42-53.

6. Gross A M J, D'Sylva J, Manipulation or Mobilisation for Neck Pain : The Cochrane Library. John Wiley \& Sons, Ltd.2010: CD004249-CD004249.

7. Rodriguez-Sanz J, Malo-Urries M, Corral-de-Toro J, Lopez-de-Celis C, Lucha-Lopez M O, Tricas-Moreno J M, Lorente A I, et al., Does the Addition of Manual Therapy Approach to a Cervical Exercise Program Improve Clinical Outcomes for Patients with Chronic Neck Pain in Short- and Mid-Term? A Randomized Controlled Trial. Int J Environ Res Public Health, 2020, 17(18).

8. Gonzalez-Rueda V, Hidalgo-Garcia C, Rodriguez-Sanz J, Bueno-Gracia E, Perez-Bellmunt A, RodriguezRubio P R,Lopez-de-Celis C, Does Upper Cervical Manual Therapy Provide Additional Benefit in Disability and Mobility over a Physiotherapy Primary Care Program for Chronic Cervicalgia? A Randomized Controlled Trial. Int J Environ Res Public Health, 2020, 17(22).

9. Gattie E, Cleland J A, Pandya J,Snodgrass S, Dry Needling Adds No Benefit to the Treatment of Neck Pain: A Sham-Controlled Randomized Clinical Trial With 1-Year Follow-up. J Orthop Sports Phys Ther, 2021, 51(1):37-45.

10. Huang J F, Zheng X Q, Chen D, Lin J L, Zhou W X, Wang H, Qin Z, et al., Can Acupuncture Improve Chronic Spinal Pain? A Systematic Review and Meta-Analysis. Global Spine J, 2021, 11(8):12481265.

11. Patel K, A G,N G, Massage for mechanical neck disorders : Cochrane Database Syst Rev. 2012: CD004871. 
12. Cavalcanti I F, Antonino G B, Monte-Silva K K D, Guerino M R, Ferreira A P L,das Gracas Rodrigues de Araujo M, Global Postural Re-education in non-specific neck and low back pain treatment: A pilot study. J Back Musculoskelet Rehabil, 2020, 33(5):823-828.

13. Pillastrini P, Banchelli F, Guccione A, Di Ciaccio E, Violante F S, Brugnettini M,Vanti C, Global Postural Reeducation in patients with chronic nonspecific neck pain: cross-over analysis of a randomized controlled trial. Med Lav, 2018, 109(1):16-30.

14. Pillastrini P, de Lima E S R F, Banchelli F, Burioli A, Di Ciaccio E, Guccione A A, Villafane J H, et al., Effectiveness of Global Postural Re-education in Patients With Chronic Nonspecific Neck Pain: Randomized Controlled Trial. Phys Ther, 2016, 96(9):1408-16.

15. Plessas A,Bernardes D, The role of ergonomic saddle seats and magnification loupes in the prevention of musculoskeletal disorders. A systematic review. Int J Dent Hyg, 2018, 16(4):430-440.

16. Myers T W, Anatomy Trains - Myofascial Meridians of manual and movement Therapy (4th Edition). Paris: Elsevier.2020: 1-360.

17. Liu C Y, Application of human body movement patterns and Kinect chain in sports. Journal of Chengdu Sport University, 2016, 42(6):1-11.

18. Ellenbecker T S, D C M,Derosa C, Effective functional progressions in sport rehabilitation : Human Kinetics.2009.

19. Yip C H T, Chiu T T W,Poon A T K, The relationship between head posture and severity and disability of patients with neck pain. Man Ther, 2008, 13(2):148-54.

20. Ghamkhar L,Kahlaee A H, Is forward head posture relevant to cervical muscles performance and neck pain? A case-control study. Braz J Phys Ther, 2019, 23(4):346-354.

21. Yu Q, Huang H, Zhang Z, Hu X, Li W, Li L, Chen M, et al., The association between pelvic asymmetry and non-specific chronic low back pain as assessed by the global postural system. BMC Musculoskelet Disord, 2020, 21(1):596.

22. Fernandez-de-las-Penas C, Alonso-Blanco C, Cuadrado M L, Gerwin R D,Pareja J A, Trigger points in the suboccipital muscles and forward head posture in tension-type headache. Headache, 2006, 46(3):454-60.

23. Richards K V, Beales D J, Smith A L, O'Sullivan P B,Straker L M, Is Neck Posture Subgroup in Late Adolescence a Risk Factor for Persistent Neck Pain in Young Adults? A Prospective Study. Phys Ther, 2021, 101(3).

24. Loudon J, Jenkins W,Loudon K, The relationship between static posture and ACL injury in female athletes. J Orthop Sports Phys Ther, 1996, 24(2):91-7.

25. Javanshir K, Rezasoltani A, Mohseni-Bandpei M A, Amiri M, Ortega-Santiago R,Fernandez-de-LasPenas C, Ultrasound assessment of bilateral longus colli muscles in subjects with chronic bilateral neck pain. Am J Phys Med Rehabil, 2011, 90(4):293-301.

26. Gill S, Postural disorders and musculoskeletal dysfunction diagnosis, prevention, treatment : Elsevier LLC.2014: 18-19. 
27. Zheng C, CH H,Wei H, Introduction of the knee's biomechanism. Chin J Orthop, 2006, 26(12):864-862.

28. Silva A G,Johnson M I, Does forward head posture affect postural control in human healthy volunteers?. Gait Posture, 2013, 38(2):352-3.

\section{Figures}

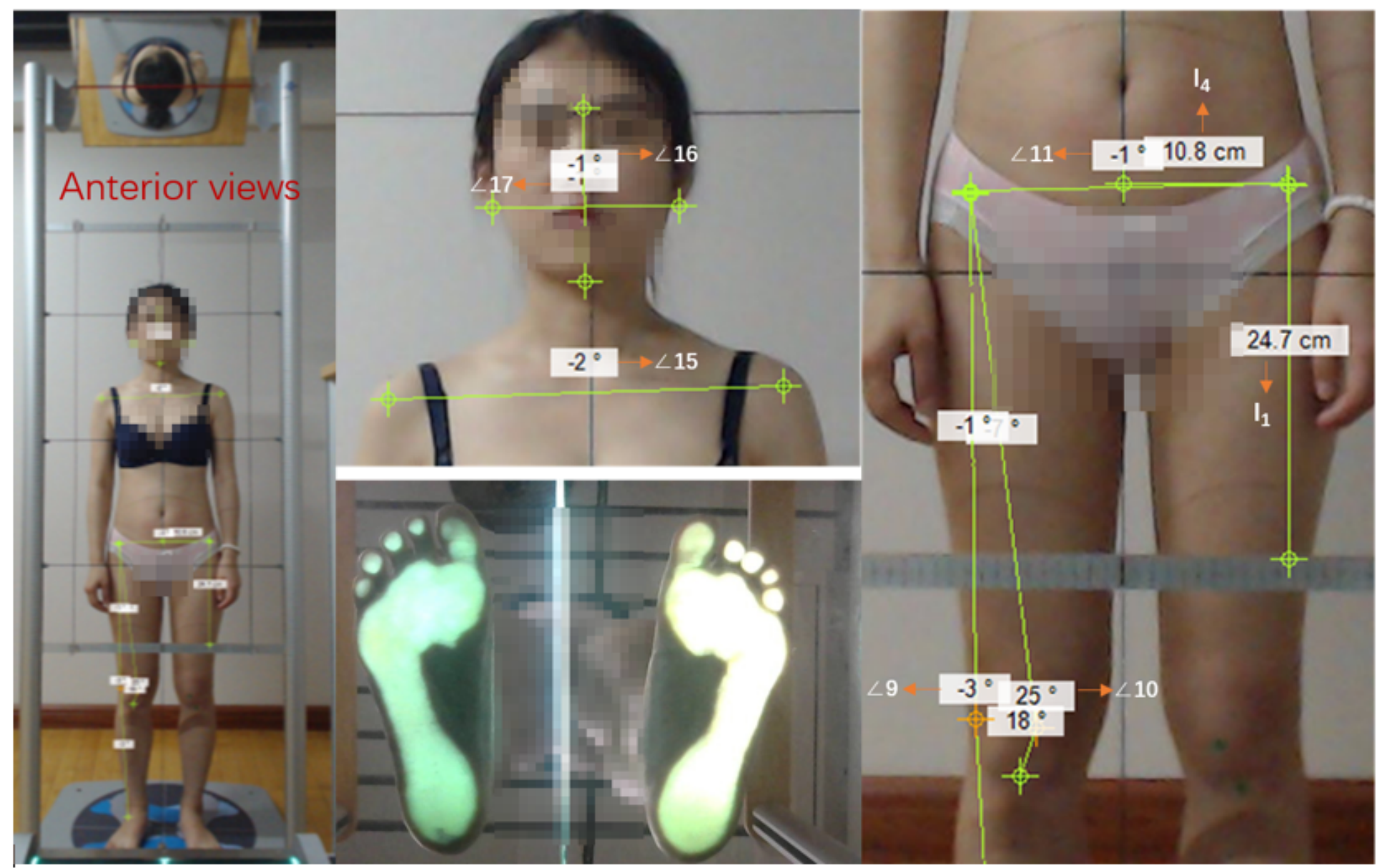

Figure 1

Anterior views 


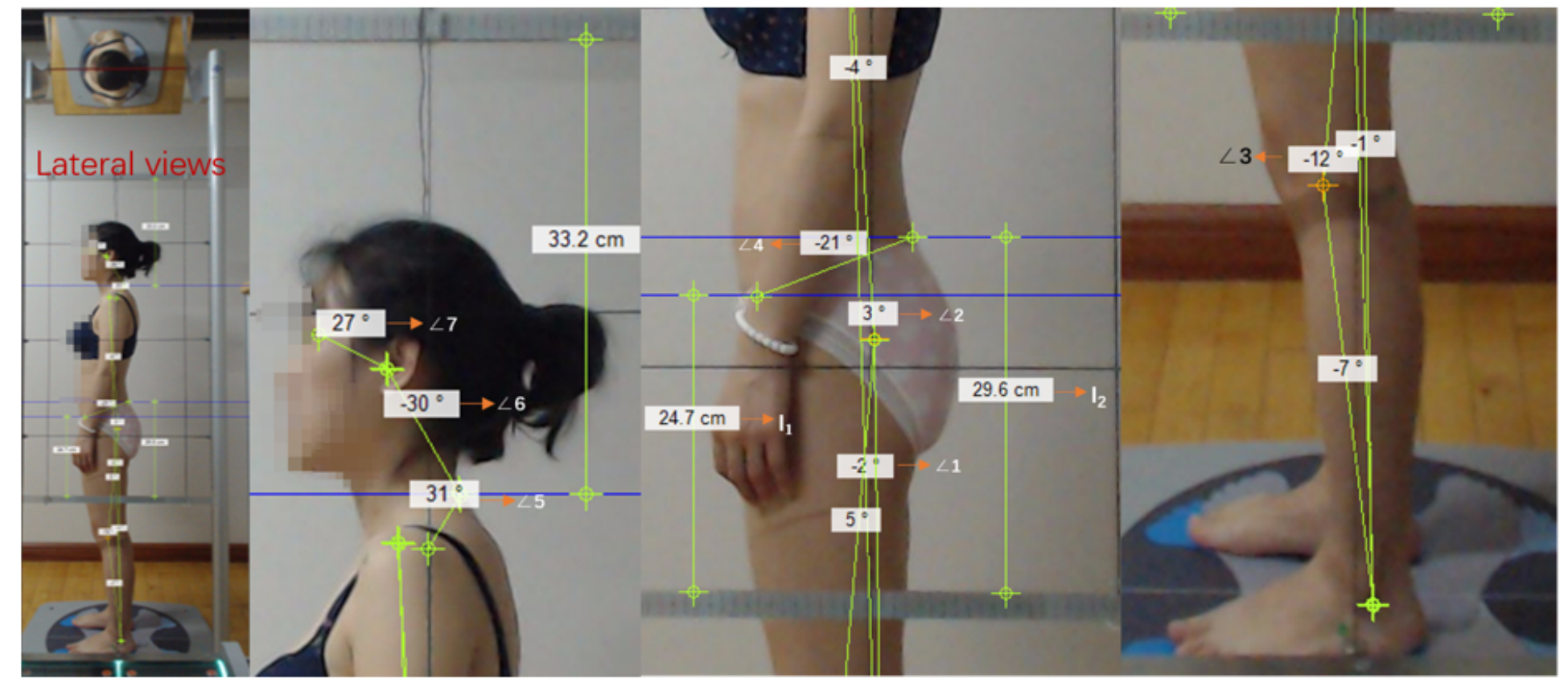

\section{Figure 2}

\section{Lateral views}

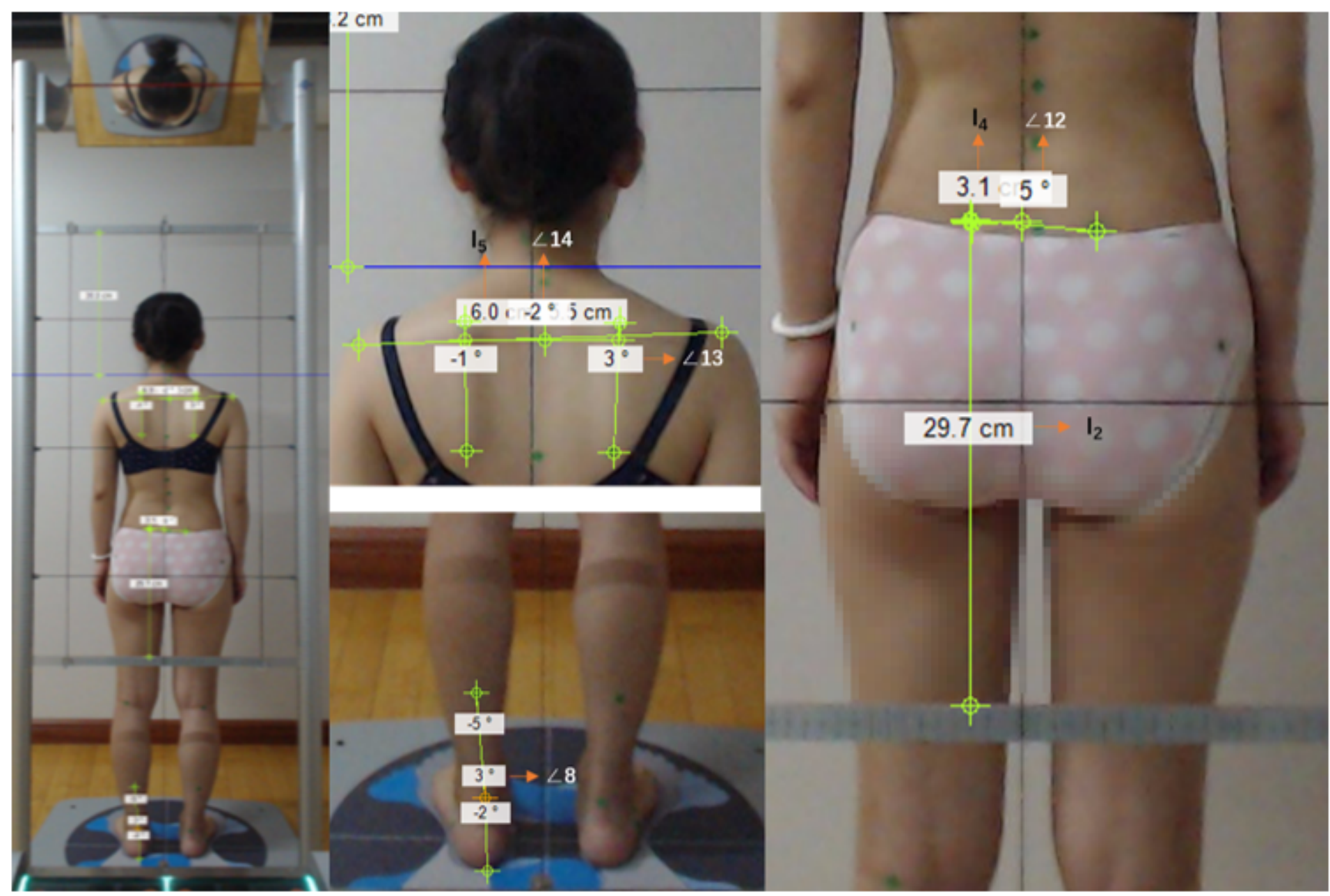

Figure 3 


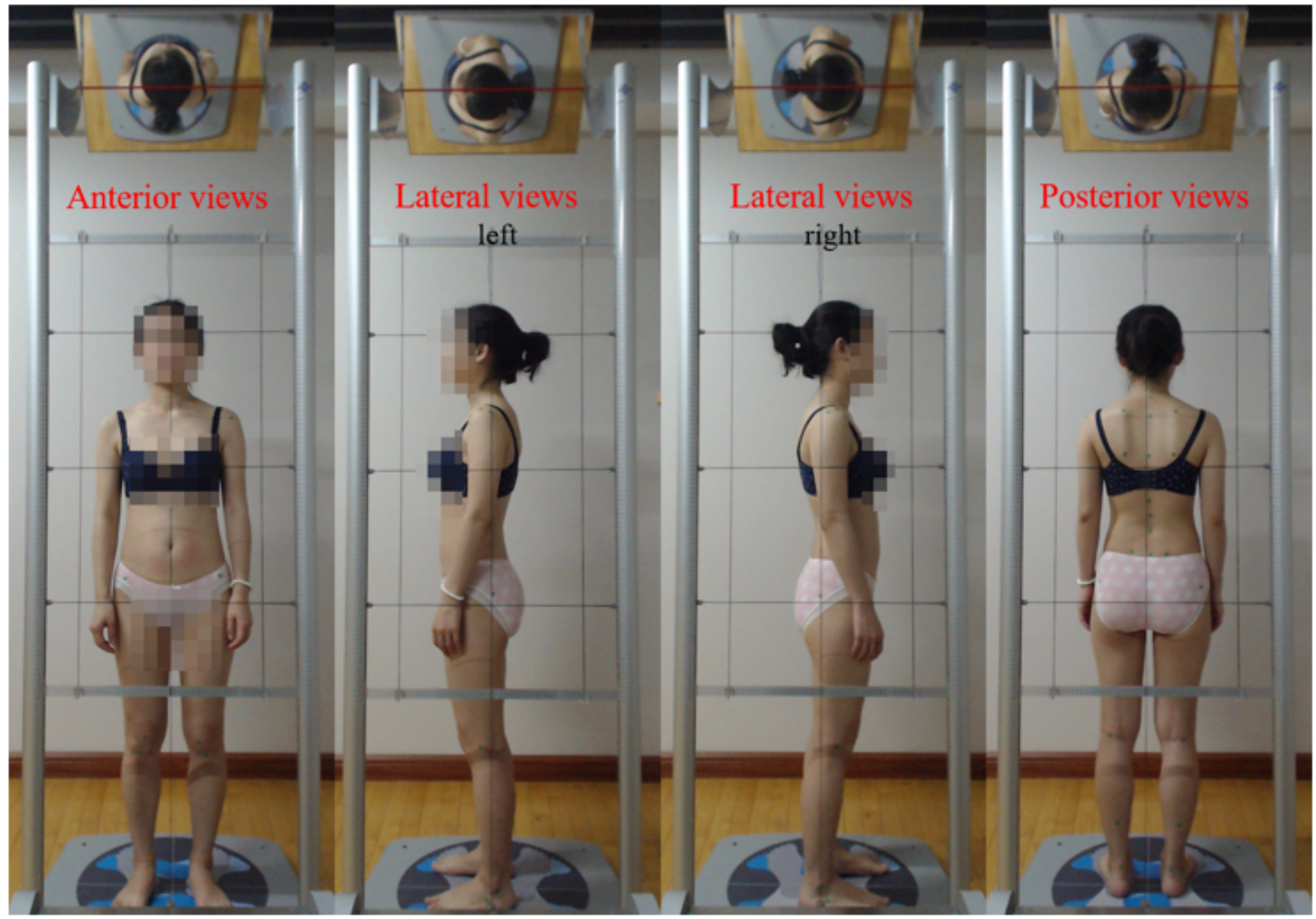

Figure 4

Photos of posture

\section{Supplementary Files}

This is a list of supplementary files associated with this preprint. Click to download.

- Supplementary1.docx

- Supplementary2.docx 